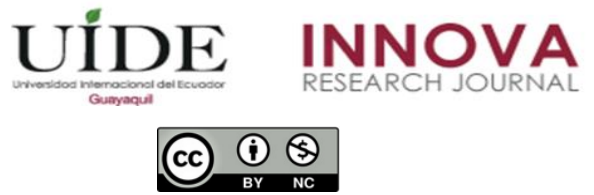

INNOVA Research Journal, ISSN 2477-9024

(Enero - Abril 2021). Vol. 6, No.1 pp. 111-128

DOI: https://doi.org/10.33890/innova.v6.n1.2021.1463

URL: http://revistas.uide.edu.ec/index.php/innova/index

Correo: innova@uide.edu.ec

\title{
Estrategia metodológica basada en tecnologías de la información y comunicación en expresión oral del idioma inglés
}

\section{Methodological strategy based on information and communication technologies in oral expression of the english language}

Silvana Patricia Meneses-Paucar

(D) https://orcid.org/0000-0002-2222-5353

Unidad Educativa Semillas de Vida, Latacunga-Ecuador

Ricardo Patricio Medina-Chicaiza

(D) https://orcid.org/0000-0002-2736-8214

Pontificia Universidad Católica del Ecuador sede Ambato, Ecuador

Autor para correspondencia: pmedina@pucesa.edu.ec; ricardopmedina@uta.edu.ec, semillas_vida@yahoo.ec

Fecha de recepción: 06 de julio de 2020 - Fecha de aceptación: 15 de octubre de 2020

\section{Resumen}

La investigación tiene por objeto desarrollar una estrategia metodológica basada en las Tecnologías de la Información y Comunicación para la mejora de la expresión oral del idioma inglés, en estudiantes del nivel de básica superior entre 14 y 15 años de la zona urbana Latacunga - Ecuador. La metodología aplicada en la investigación se sustenta en la recopilación de información documental y bibliográfica indagada bajo la herramienta tecnológica Perish, los métodos son síntesis - análisis, inductivo - deductivo, con un diagnóstico en base a la técnica de observación directa y encuestas a docentes y estudiantes analizados, la confiabilidad de los instrumentos se realizó a través de Alpha de Cronbach, todo esto para alcanzar un conocimiento profundo acerca de las principales características del tema de estudio. Dentro de los resultados se propone una estrategia metodológica basada en las tecnologías de la información y comunicación con las siguientes fases: Diagnóstico, Planificación, Aplicación y Control, así también, se determina la importancia de la expresión oral en el entorno educativo como en otros contextos y la relevancia del uso de herramientas tecnológicas para establecer un aprendizaje dinámico, atractivo, acorde a las necesidades educativas de una población estudiantil actual.

Palabras claves: estrategia metodológica; tecnología de la información y comunicación; expresión oral; inglés; idioma extranjero.

\footnotetext{
Abstract

The objective of the research is to develop a methodological strategy based on Information and Communication Technologies for the improvement of the oral expression of English language, in students of the upper basic level with ages between 14 and 15 years in the area Latacunga -
} 
Ecuador. The methodology applied in the research is based on the documental compilation and bibliographic information investigated under the Perish technological tool, the methods are synthesis-analysis, inductive-deductive, with a diagnosis based on direct observation technique and surveys to teachers and students. The reliability of the instruments was carried out through Cronbach's Alpha, all this to achieve a deep knowledge about the main characteristics of the study topic. Within the results, the author proposes a methodological strategy based on information and communication technologies with the following phases: Diagnosis, Planning, Application and Control, as well as, determining the importance of oral expression in the educational environment as in other contexts and the relevance of the use of technological tools to establish dynamic, attractive learning, according to the educational needs of a current student population.

Keywords: methodological strategy; information and communication technologies; oral expression; english; foreign language.

\section{Introducción}

El idioma en una nación representa el medio de comunicación por el cual los miembros que lo conforman se relacionan y comunican; esto se ve ligado a la diversidad propia de su cultura. Por sus características, se reconoce que cada espacio mantiene un lenguaje natal que, de cierta forma, no se constituye como el elemento único de comunicación, pues desde la antiguiedad y con los diversos sistemas de movilidad, el ser humano se ha vinculado a medios de expresión extranjeros. Es allí, donde el inglés ha tomado mayor auge entre los dialectos que se hablan alrededor del mundo por tratarse de un idioma universal que rompe fronteras y mantiene la denominación como lengua franca (Seidlhofer, 2001). Por tanto, se ha utilizado por hablantes que no mantienen al inglés como primera lengua sino que optan porque sea el canal para hacerse entender, establecer relaciones, interactuar con información e incluso aprender.

Según Vergara y Perdomo (2017) señalan que, el inglés es el idioma más usado para la comunicación internacional y hablarlo representa una ventaja cultural, social y laboral, pues, la lengua extranjera forma parte de todos los ámbitos. Se asume entonces, que una persona preparada mantiene mayores oportunidades, y es aquí, donde su aprendizaje marca importancia en la vida de estas personas.

En sintonía con el párrafo anterior, (Nunan, 1991;Golkova y Hubackova, 2014;Martínez, 2020) coinciden que, el dominio del idioma inglés en especial la expresión oral (speaking), requiere mayor energía por tratarse de una necesidad humana que satisface la comunicación de quienes hablan o desean hablar la lengua inglesa. Adquirir el speaking en países hispanohablantes necesita de una serie de carácterísticas, mismas que promueven alcanzar su dominio, los establecimientos educativos junto a sus docentes son los encargados en promover la práctica de esta habilidad; sin duda alguna, el nivel del dominio del speaking marca una ventaja de crecimiento personal a quienes lo han desarrollado.

En el Ecuador, según el Ministerio de Educación (2014), en su Acuerdo No 052-14, artículo 1, expone que, la enseñanza del inglés a partir del año lectivo 2016-2017 régimen Sierra y 2017-2018 régimen Costa, sea obligatoria desde el segundo grado de Educación General Básica (EGB) hasta el tercer curso de Bachillerato para todas las instituciones públicas, 
fiscomisionales y particulares del país. Por tanto, se reconoce como una asignatura de vital importancia para su aprendizaje dentro de la malla currillar de los estudiantes.

Uno de los estudios realizados en el país por Education First (2019), señala en sus resultados que, el dominio de las habilidades del inglés es un elemento que posiciona la clasificación del ranking. Es así, que se estableció un examen a 950.000 estudiantes de 100 países alrededor del mundo, en donde el Ecuador alcanzó el puesto número 81, constituyendo su orden a partir de los países denominados más altos por mayor competencia en inglés, hasta los más bajos.

Ante ello, la adquisición y dominio de la expresión oral de un segundo idioma no debe tomarse como algo a la ligera, pues se requiere de la participación de las Tecnologías de la Información y Comunicación (TIC), así lo manifiesta Yorca et al (2019), ya que estas ayudan a los educandos a aprender así como a los pedagogos de estas áreas les permite enseñar, de tal manera que sea atractiva y eficiente, pues para la educación existe una gama amplia de herramientas que desde años atrás se encuentran presentes.

Para (Gonzales et al., 2017; Vélez y Yaguana, 2019), hablar de un proceso de enseñanza aprendizaje en donde no se encuentre inmersas las tecnologías de la información y comunicación es algo casi imposible, pues estas se definen como el aporte que no solo perfecciona un proceso educativo, sino que es el camino para un cambio cultural a favor de la calidad, en donde se minimiza el tiempo y evita procesos mecánicos, ante esto, es importante mencionar que esta apreciación recalca que el uso de las TIC's debe estar inmerso en las actividades escolares, pues su contribución es significativa a favor de la educación.

Al respecto Ricoy y Álvarez (2016), exponen que los obstáculos evidenciados, a pesar de contar con una variedad de instrumentos, se basan en la ausencia de metodologías aplicadas para el alumnado, a lo que acompaña Saettler (1990), quien manifiesta que el buen uso y enfoque de las herramientas tecnológicas manipuladas por los docentes no deben centrarse en la moda de usar nuevas tecnologías, sino enfocarse en el fin que tienen estas para ser parte del proceso educativo; en este sentido, alineado a mejorar las habilidades lingüísticas de un idioma propulsor de desarrollo.

Es así, que a través de la observación directa se identifica la situación problemática en una Institución de Educación General Básica (EGB) en la zona urbana de Latacunga - Ecuador, en donde jóvenes de edades promedio entre 14 y 15 años, enfrentan debilidades en el desarrollo de la expresión oral cuya consecuencia es el bajo rendimiento; además, en los docentes se evidencia la ausencia de estrategias metodológicas con el uso de las TIC's que apoyen a esta generación $\mathrm{Z}$ que requiere una educación activa.

Entre los beneficiarios directos de este proyecto investigativo están los estudiantes y docentes de EGB, así como de manera indirecta los padres de familia, autoridades y comunidad educativa, pues se propone como objetivo desarrollar una estrategia metodológica basada en las tecnologías de la información y comunicación para la mejora de la expresión oral en el idioma inglés. 


\section{Metodología}

El trabajo investigativo se fundamentó en la herramienta tecnológica Perish (Harzing, 2016), la cual facilitó la búsqueda bibliográfica en Google Académico y Google Libros para recopilar información de artículos científicos sobre el objeto de estudio: estrategias metodológicas y expresión oral.

Los métodos teóricos utilizados fueron cuadros de análisis-síntesis inductivo deductivo. Para los métodos empíricos, se aplicó la observación directa con una rúbrica de valoración de los componentes de la oralidad. La población total la constituyeron 3 docentes y 41 estudiantes pertenecientes al nivel de básica superior.

El análisis de documentos y encuestas en línea se estableció a los mismos docentes y estudiantes, en base a 11 preguntas cerradas y a través de una herramienta electrónica en el formulario de Google Drive. Es necesario señalar que la confiabilidad de los instrumentos aplicados se validaron con la ayuda del Programa Estadístico SPSS, a través de Alpha de Cronbach en la cual se obtuvo 0.887 , valor que garantiza fiabilidad al instrumento aplicado, allí se detalló aspectos como el uso de estrategias metodológicas en el salón de clases, aplicación de TIC's en la expresión oral, frecuencia de actividades enfocadas al Speaking; situación que permitió palpar las características del problema en relación a la habilidad oral, corroborándose con el diagnóstico de los estudiantes.

\section{Conceptos iniciales}

\section{El software educativo en la enseñanza del idioma inglés}

Hoy en día, se comprende al software educativo como una herramienta pedagógica que ayuda a la adquisición de conocimientos y al desarrollo de habilidades, conforme a (Azurra, 2015; Núñez y Medina, 2020), son programas de carácter didáctico usados en el contexto de la enseñanza aprendizaje, es decir que se encuentran dentro del ámbito escolar, allí se aprecia una serie de softwares disponibles, muchos de estos adaptados como apoyo a la docencia y otros con la finalidad de orientar al alumno; ambos contribuyen al proceso educativo de manera autónoma y colaborativa.

Conforme a Maldonado et al (2020), las transformaciones significativas que consienten a las tecnologías se basan en la gran acogida que estos han tenido en diferentes áreas, pero de manera específica en la educación, a través de los softwares educativos, convirtiéndose en elementos necesarios de la vida cotidiana de los educandos; es decir, son aplicables a todos los niveles instructivos, favorecen un cambio en los aprendizajes de los estudiantes a través de la mejora de los conocimiento y habilidades cognitivas.

Una apreciación similar es la que comparte Vidal et al (2010), el software educativo en la actualidad se ha convertido en el instrumento imprescindible para los procesos de enseñanza aprendizaje, pues se conoce que las exigencias de la sociedad actual, se alinean cada vez más a la parte informática y a los saberes tecnológicos; es así, que se puede manifestar que quién no se 
encuentra a la par de estos conocimientos pudiera estar perdiéndose de la contribución que estos brindan al desarrollo educativo.

A continuación se presenta una categorización, en la cual según Pacheco y Barros (2013) abordan las características y usos de las herramientas tecnológicas dentro del proceso de aprendizaje, siendo estas:

Plataformas y cursos en línea: Creado para el desarrollo de cursos o módulos didácticos en la web, a través del uso de las tecnologías de la información y comunicación tanto de manera sincrónica como asincrónica. Su principal desventaja se establece en que la mayoría de estas requieren una cancelación para su uso.

Aplicación o apoyo: Programas creados sin un fin específicamente educativo, pero que pueden aplicarse al uso escolar, en esta categoría están los procesadores de texto, hojas de cálculo o bases de datos.

Ejercicios y prácticas: Aplicado para revisar lecciones de contenidos y ejercicios evaluativos, para su uso se requiere que los usuarios cuenten con conocimientos; su fin se determina en aplicar o retroalimentar los conocimientos.

Tutoriales: Breves y de escasa profundidad, contienen información didáctica secuenciada sobre las características principales de algún elemento o tema. Su modo es explicativo, permite revisar y repasar el material las veces que sean necesarias, aunque la participación del usuario es limitada a leer o escuchar información.

Juegos educativos: Incluye temáticas con elementos didácticos que permiten interactuar, desafiar o competir al mismo tiempo que alcanzar conocimientos, se requiere de una adecuada selección, pues, algunos podrían desviarse de su fin.

Multimedia: Espacio propicio para buscar e interactuar con información; contiene un sinnúmero de elementos, entre estos: medios gráficos, físicos y digitales, los cuales posibilitan un aprendizaje enfocado a las inteligencias múltiples, en donde su infinidad de presentaciones cubre la forma de adquirir, practicar o retroalimentar conocimientos de los individuos. Es necesario señalar que requieren conexión a red para ser aplicados.

En uno de los elementos de este grupo de software educativo se destacará al servicio en línea Vocaroo, por ser el instrumento seleccionado a usarse en el diseño de la propuesta de este trabajo investigativo para la mejora de la expresión oral, el cual según Abio (2014), es una aplicación de fácil acceso que tiene por objeto grabar la voz de manera clara, se guarda y comparte en diversos formatos en tiempo récord; es decir que, a través de esta, se lleve a cabo prácticas en el desarrollo de la habilidad comunicativa; además, sea accesible y no solicite registro alguno.

Una vez enunciada esta herramienta se presenta un cuadro de análisis de su importancia: 


\section{Tabla 1}

Análisis de Vocaroo con otras herramientas

\begin{tabular}{|c|c|c|c|c|}
\hline CARACTERÍSTICAS & Flipgrid One & $\begin{array}{l}\text { Flipgrid } \\
\text { Classroom }\end{array}$ & Duolinguo & Vocaroo \\
\hline Tipo & Plataforma gratuita & $\begin{array}{l}\text { Plataforma } \\
\text { pagada }\end{array}$ & Plataforma gratuita & Servicio en línea \\
\hline Aplicable en & $\begin{array}{l}\text { Monitor, Teléfono } \\
\text { móvil }\end{array}$ & $\begin{array}{l}\text { Monitor, } \\
\text { Teléfono móvil }\end{array}$ & $\begin{array}{l}\text { Monitor, Teléfono } \\
\text { móvil }\end{array}$ & $\begin{array}{l}\text { Monitor y Teléfono } \\
\text { móvil }\end{array}$ \\
\hline Tiempo de práctica & $\begin{array}{l}90 \text { segundos } \\
1 \text { práctica }\end{array}$ & $\begin{array}{l}90 \text { segundos } \\
\text { Prácticas } \\
\text { ilimitadas }\end{array}$ & $\begin{array}{l}\text { Prácticas limitadas } \\
\text { Fases diarias }\end{array}$ & Prácticas ilimitadas \\
\hline $\begin{array}{l}\text { Habilidad que } \\
\text { desarrolla }\end{array}$ & Speaking & Speaking & $\begin{array}{l}\text { Speaking } \\
\text { Writing } \\
\text { Listening }\end{array}$ & Speaking \\
\hline $\begin{array}{l}\text { Requiere registrar } \\
\text { cuenta }\end{array}$ & $\mathrm{Si}$ & $\mathrm{Si}$ & $\mathrm{Si}$ & No \\
\hline Incluir contraseña & $\mathrm{Si}$ & $\mathrm{Si}$ & $\mathrm{Si}$ & No \\
\hline $\begin{array}{l}\text { Solicita información } \\
\text { previa a la práctica }\end{array}$ & $\mathrm{Si}$ & $\mathrm{Si}$ & $\mathrm{Si}$ & No \\
\hline Usa Video & $\mathrm{Si}$ & $\mathrm{Si}$ & No & No \\
\hline Configuración excesiva & $\mathrm{Si}$ & $\mathrm{Si}$ & No & No \\
\hline $\begin{array}{l}\text { Modalidad para } \\
\text { compartir }\end{array}$ & $\begin{array}{l}\text { Enlace } \\
\text { Enlace HTML } \\
\text { Facebook } \\
\text { Twitter } \\
\text { Código Qr }\end{array}$ & $\begin{array}{l}\text { Enlace } \\
\text { Enlace HTML } \\
\text { Facebook } \\
\text { Twitter } \\
\text { Código Qr }\end{array}$ & $\begin{array}{l}\text { No se comparte } \\
\text { mantiene registro } \\
\text { de las actividades } \\
\text { dentro de la } \\
\text { plataforma }\end{array}$ & $\begin{array}{l}\text { Enlace } \\
\text { Enlace HTML } \\
\text { Formato MP3 } \\
\text { Facebook } \\
\text { Twitter } \\
\text { Reddit } \\
\text { Whatsapp } \\
\text { E-mail } \\
\text { Código QR }\end{array}$ \\
\hline
\end{tabular}

Fuente: elaboración propia

\section{El aprendizaje del idioma inglés}

Para las personas, el aprendizaje de un idioma representa una oportunidad de progreso. $\mathrm{Si}$ se habla del idioma inglés, este es necesario, pues se ha convertido en la línea de comunicación entre los países que no mantienen el mismo lenguaje. Ahí la importancia de su preparación, en donde, se requiere la disposición del educador para compartir, orientar e interactuar con el idioma, un educando motivado, interesado y participativo dentro del proceso, pero sobre todo una estrategia o actividades bien consolidadas que refuerce el desarrollo de la habilidad comunicativa.

Ante ello, el autor establece una revisión a la literatura de las múltiples actividades que se utilizan para la adquisición del inglés como lengua extranjera (Ver tabla 2). 


\section{Tabla 2}

\section{Revisión de la literatura sobre el aprendizaje del idioma según autores}

\begin{tabular}{|c|c|c|c|}
\hline Autor & Año & Estrategia & Metodología \\
\hline Costa & 2007 & $\begin{array}{l}\text { Programas } \\
\text { multimedia }\end{array}$ & $\begin{array}{l}\text { 1. Establecer una evaluación inicial para reconocer las } \\
\text { necesidades del grupo. } \\
\text { 2. Integrar los programas multimedia conforme a los } \\
\text { contenidos que se encuentran en el currículo. } \\
\text { 3. Usar los programas multimedia. } \\
\text { 4. Evaluar la utilidad de los programas conforme a la } \\
\text { temática. }\end{array}$ \\
\hline
\end{tabular}

Observación: Las herramientas multimedia se aplicaron para la adquisición de las cuatro destrezas del idioma inglés, este trabajo permitió un desarrollo práctico, dinámico e incluso superó el tiempo y la distancia. La metodología que compone esta investigación se encuentra inmersa en la lectura. Una de las dificultades que se presenta denota en el acceso a la red para la ejecución de los programas de multimedia.

\begin{tabular}{lll}
\hline Marín & 2009 & Proceso \\
& Tradicional
\end{tabular}

1. Presentar frases de acceso común para espacios como inicio, desarrollo y finalización a una conversación.

2. Incentivar asumir riesgos en la ejecución o práctica, sin considerar los errores que puedan presentarse en el camino.

3. Motivar a la no aplicación de estrategias comunicativas como uso de mímicas y gestos, palabras en español, extranjerismos o aproximaciones comúnmente en el desarrollo de la oralidad.

4. Efectuar oportunidades de prácticas entre pares o equipos.

Observación: El proceso efectuado se encuentra cimentado en una estrategia tradicional, cada paso es representado en ejercicios básicos, no existe la colaboración de ningún tipo de recurso pues accede solo a la voz como el instrumento de práctica. Está centrado en el desarrollo de la destreza comunicativa con un alto grado de práctica, los aspectos débiles se vinculan a la falta de dinamismo y motivación que presenta para los estudiantes al no contar con materiales que capturen su atención o sean novedosos.

$\begin{array}{lll}\text { Chacón \& Clevia } 2010 & \text { Podcast } & \text { 1. Iniciar la etapa de entrenamiento. } \\ \text { J. Pérez } & & \text { 2. Planificar los contenidos y el tema del podcast. } \\ & \text { 3. Realizar la producción del podcast. } \\ & \text { 4. Efectuar el montaje y la publicación. } \\ & \text { 5. Evaluar los hallazgos tras su aplicación. }\end{array}$

Observación: El acceso al internet es un punto clave para su aplicación y publicación, es de gran aporte para la expresión oral, su adecuada práctica efectiviza los procesos de aprendizaje y este puede ser autónomo, las desventajas de este se deben a exageradas configuraciones.

\begin{tabular}{lll} 
Utrera & Juegos & 1. Planificar los contenidos apoyado en el juego. \\
& & 2. Considerar los instrumentos o materiales. \\
& & 3. Llevar a cabo los juegos de manera organizada. \\
& $4 . \quad$ Reforzar los contenidos durante su ejecución. \\
\hline
\end{tabular}

Observación: La participación del inglés a través de los juegos permite el desarrollo de la creatividad, la sana diversión y el aprendizaje espontáneo. Su ejecución se alineó al mejoramiento del vocabulario, pronunciación y al mayor contacto con el idioma inglés. Para su aplicación el uso de recursos básicos como juguetes, material concreto y música han generado el libre acceso para su práctica, aquí su desventaja es desviarse del fin educativo por falta de reglas claras. 


\begin{tabular}{|c|c|c|c|}
\hline Autor & Año & Estrategia & Metodología \\
\hline Toscano \& & 2013 & La música & Mantener un acercamiento melódico. \\
\hline Fonseca & & & Planificar los contenidos con el uso de canciones. \\
\hline & & & Desarrollar rimas, rondas o canciones. \\
\hline & & & 4. Establecer prácticas con la música. \\
\hline & & & 5. Evaluar la adquisición del lenguaje. \\
\hline
\end{tabular}

Observación: La aplicación de la inteligencia musical o alguno de sus elementos sonoros mantiene como beneficio el aspecto lingüístico, sociolingüístico y afectivo, pues mejora la oralidad, la comprensión lectora, la asimilación, recuperación de léxico, estructura gramatical y la motivación. Por otro lado, como dificultad presenta la falta de interés en ciertos grupos, pues es de mayor aceptación en infantes.

\begin{tabular}{|c|c|c|c|}
\hline $\begin{array}{l}\text { Pizarro \& } \\
\text { Cordero }\end{array}$ & 2013 & $\begin{array}{l}\text { Entornos } \\
\text { digitales } \\
\text { Y } \\
\text { Sistemas } \\
\text { multimedia }\end{array}$ & $\begin{array}{l}\text { 1. Planear las temáticas a desarrollar. } \\
\text { 2. Seleccionar las actividades que focalizarán de } \\
\text { manera clara y efectiva los temas. } \\
\text { 3. Priorizar los recursos que manejan los estudiantes } \\
\text { diariamente. } \\
\text { 4. Utilizar dichos recursos. }\end{array}$ \\
\hline
\end{tabular}

Observación: La aplicación de estos recursos permite una metodología comunicativa; su forma de aprender ha ganado espacios por denominarse innovadora y atractiva, así como por superar el tiempo y espacio. El acceso al internet, así como la tensión ante el desconocimiento de quien cumple el rol de facilitador, se han convertido en las barreras de esta actividad.

\begin{tabular}{|c|c|c|c|}
\hline Morales et al. & 2015 & $\begin{array}{l}\text { Realidad } \\
\text { Aumentada }\end{array}$ & $\begin{array}{l}\text { 1. Establecer una conexión entre el equipo y el } \\
\text { programa de realidad aumentada. } \\
\text { 2. Verificar las temáticas con las que cuenta el } \\
\text { programa, en caso de ausencia construir el } \\
\text { contenido. } \\
\text { 3. Aplicar realidad aumentada. }\end{array}$ \\
\hline
\end{tabular}

Observación: Se desarrolla a través de un móvil, el cual es de fácil acceso, portable y al alcance de la población estudiantil. Al ser una estrategia llamativa captura la atención de los estudiantes, dando resultados de un aprendizaje eficaz. Utiliza tres de los cinco sentidos.

Como dificultad se presenta el desconocimiento por parte de los docentes para desarrollar temáticas, pues demanda de una programación previa.

$\begin{array}{ll}\text { Gavarri Duolingo } 2016 & \begin{array}{l}\text { 1. Realizar el registro en la plataforma. } \\ \end{array} \\ & \text { 2. Desarrollar la práctica con temas previamente } \\ \text { aprendidos. }\end{array}$

Observación: El uso de la plataforma faculta una cultura digital, en donde el horario y lugar no son inconvenientes, pues el aprendizaje se tiene a la mano, esta plataforma es gratuita y permite llevar a cabo destrezas como escuchar, leer, escribir, ciertamente no tiene mayor contribución a la destreza oral. Conforme al autor esta no permite un progreso de los aprendices iniciales, pues se maneja como una herramienta para afianzar conocimientos.

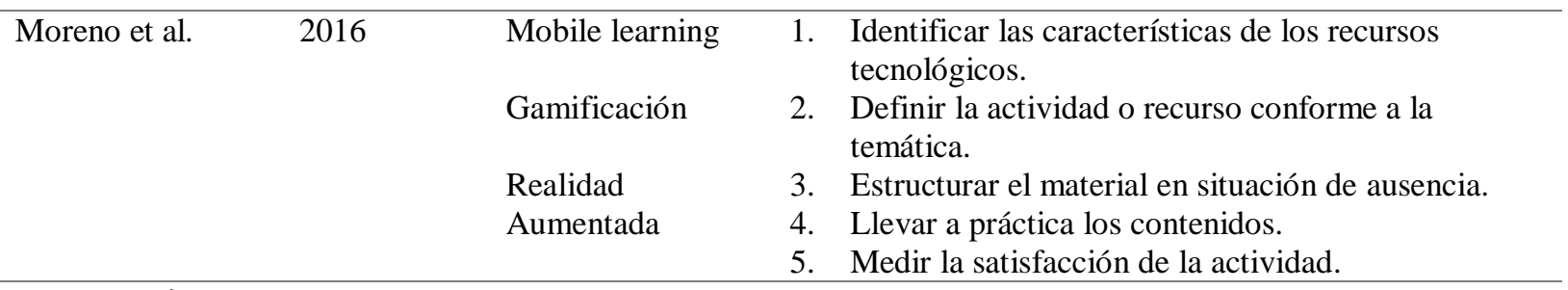

Observación: La aplicación de esta estrategia se encuentra centrada en las cuatro destrezas del idioma inglés, mantiene énfasis en determinar un avance en las necesidades de la actual población digital, es considerada novedosa, práctica, motivadora e influyente, pues propone un incremento en la creatividad y la producción del 


\section{Autor Año $\quad$ Estrategia Metodología}

conocimiento. La metodología en esta investigación no se encuentra bien definida pues presenta un sin número de herramientas, que poseen características particulares. La dificultad más notable en esta actividad refiere a no contar con docentes o facilitadores con conocimiento en el uso de este tipo de TIC, quienes elaboren, modifiquen o reutilicen material informático a favor de sus contenidos.

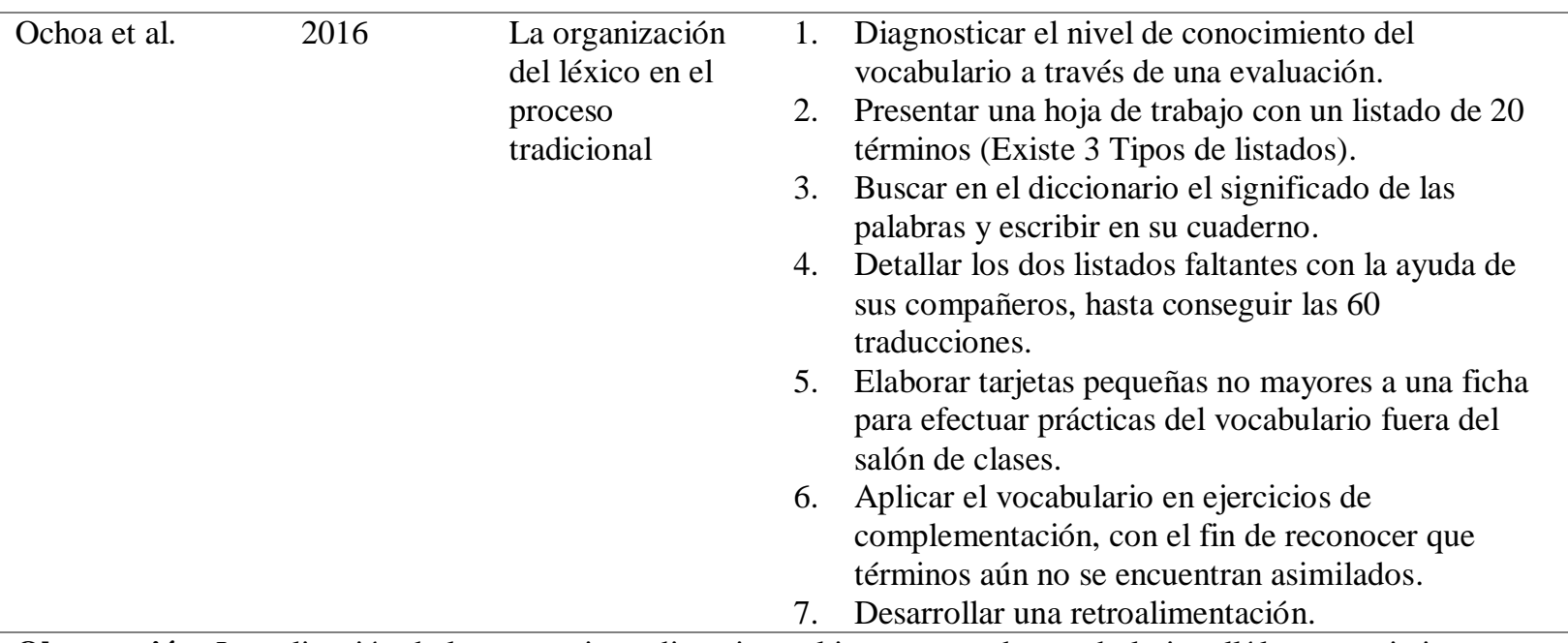

Observación: La aplicación de la estrategia se direcciona al incremento de vocabulario, allí la memoria juega un rol fundamental, pues a través de esta se adquieren más términos, motivo que desarrolla la competencia gramatical y discursiva. Inmerso en el proceso está el uso del diccionario, recursos que faculta el recordar aprender explorar y alcanzar, para la autora, el proceso que desmotiva en la aplicación de la estrategia se centra en la escasa presencia de materiales o recursos que sean atractivos y causen motivación y novedad en los estudiantes.

\begin{tabular}{|c|c|c|c|}
\hline Aldana & 2018 & $\begin{array}{l}\text { Estrategia } \\
\text { integral } \\
\text { En base a modelo } \\
\text { del ABP }\end{array}$ & $\begin{array}{l}\text { 1. Planificar contenidos. } \\
\text { 2. Elaborar material concreto. } \\
\text { 3. Escribir textos o frase para usar. } \\
\text { 4. Desarrollar lecturas propias. } \\
\text { 5. Observar video o imágenes para simular gestos y } \\
\text { diálogos. } \\
\text { 6. Preparar material para efectuar ejercicios de roles }\end{array}$ \\
\hline
\end{tabular}

Observación: La estrategia integral aplica un modelo educativo basado en el ABP para fortalecer las cuatro habilidades del idioma inglés, es decir desarrollarlas en conjunto, para su ejecución requiere que el estudiante efectué una serie de actividades como construcción del material entre estos tarjetas, textos, videos y material de preparación autónoma, situación que se convierte en desfavorable por la cantidad de actividades y el tiempo que le demanda para su elaboración.

\begin{tabular}{|c|c|c|c|}
\hline Madero & 2018 & Lúdica & $\begin{array}{l}\text { 1. Planificar los contenidos del área. } \\
\text { 2. Categorizar las actividades lúdicas conforme a la } \\
\text { temática sean por: memorización, práctica, } \\
\text { predicción o inferencia deductiva, } \\
\text { contextualización y anotaciones. } \\
\text { 3. Ejecutar los talleres en combinación del tema y la } \\
\text { categoría. } \\
\text { 4. Monitorear y evaluar su práctica. }\end{array}$ \\
\hline
\end{tabular}

Observación: Se enfoca de manera específica al desarrollo de la destreza fonológica, es decir al Speaking. Para su práctica desarrolla actividades de entrevista, karaoke, dramatizaciones individuales y grupales, canciones y videos musicales. Esta estrategia captura el interés, pues mantiene como característica el juego y la dinámica, aunque conserva un estilo tradicional de aprendizaje para los estudiantes. 


\begin{tabular}{|c|c|c|c|}
\hline Autor & Año & Estrategia & Metodología \\
\hline \multirow{7}{*}{ Norato \& López } & 2018 & Material concreto & 1. Planificar los contenidos. \\
\hline & & & 2. Realizar un diagnóstico centrándose en la \\
\hline & & * Recetas de & participación de los estudiantes. \\
\hline & & $\begin{array}{l}\text { cocina } \\
* \text { Elementos }\end{array}$ & $\begin{array}{l}\text { 3. Establecer grupos de trabajo para la construcción } \\
\text { del material concreto. }\end{array}$ \\
\hline & & $\begin{array}{l}\text { químicos con } \\
\text { material }\end{array}$ & $\begin{array}{l}\text { 4. Otorgar la guía e instrucción en la ejecución de } \\
\text { material. }\end{array}$ \\
\hline & & reciclable & $\begin{array}{l}\text { 5. Fortalecer el vocabulario con la ayuda de videos y } \\
\text { carteles. }\end{array}$ \\
\hline & & & $\begin{array}{l}\text { 6. Solicitar la exposición y desarrollo de la oralidad } \\
\text { en valoración de fluidez y pronunciación. }\end{array}$ \\
\hline
\end{tabular}

Observación: En esta estrategia la práctica del idioma inglés se vincula a otras áreas; es decir, genera la multidisciplinariedad; las temáticas usadas son situaciones de la vida real. Fortalece las habilidades de escritura y oralidad, a través del uso de vocabulario y estructura gramatical, como factor negativo se evidencia la escasa participación de elementos multimedia o recursos TIC.

\begin{tabular}{|c|c|c|c|}
\hline \multirow[t]{3}{*}{$\begin{array}{l}\text { Cevallos \& } \\
\text { Becerra }\end{array}$} & \multirow[t]{3}{*}{2019} & Dramatización & $\begin{array}{l}\text { 1. Establecer equipos de trabajo. } \\
\text { 2. Presentar una lectura sobre el tema a desarrollar } \\
\text { 3. Clasificar y distribuir la información de los } \\
\text { 4. Eersonajes. } \\
\text { 5. Preporar un bosquejo en un organizador o cuadron. } \\
\text { 6. Presentar a la audiencia. } \\
\text { 7. Evaluar el producto. }\end{array}$ \\
\hline & & $\begin{array}{l}\text { Aproximación a } \\
\text { la realidad }\end{array}$ & $\begin{array}{l}\text { 1. Seleccionar un gráfico o material. } \\
\text { 2. Formular un escrito, relato, narración o } \\
\text { descripción con la ilustración u objeto. } \\
\text { 3. Presentar ideas de manera oral al grupo. } \\
\text { 4. Mantener un refuerzo o retroalimentación. } \\
\text { 5. Evaluar bajo escala. }\end{array}$ \\
\hline & & $\begin{array}{l}\text { Uso de } \\
\text { pensamiento } \\
\text { creativo } \\
\text { divergente y } \\
\text { lateral }\end{array}$ & $\begin{array}{l}\text { 1. Mostrar una tarjeta con una clave, palabra o frase. } \\
\text { 2. Motivar la construcción de una oración, frase o } \\
\text { párrafo. } \\
\text { 3. Guiar el proceso. } \\
\text { 4. Monitorear con la ayuda de una rúbrica. }\end{array}$ \\
\hline
\end{tabular}

Observación: Las estrategias aplicadas pretenden efectivizar la destreza oral del idioma inglés, se direcciona a un proceso de aprendizaje tradicional, existe limitado uso de TIC y la participación de material concreto no es variada.

\begin{tabular}{|c|c|c|c|}
\hline Yumi et al. & 2020 & $\begin{array}{l}\text { Uso del } \\
\text { WhatsApp }\end{array}$ & $\begin{array}{l}\text { 1. Establecer un diagnóstico del nivel de habla de los } \\
\text { estudiantes. } \\
\text { 2. Presentar un Test alineado a la competencia } \\
\text { comunicativa. } \\
\text { 3. Aplicar el test con el uso de WhatsApp. } \\
\text { 4. Análisis de los resultados alcanzados. }\end{array}$ \\
\hline
\end{tabular}

Observación: Esta estrategia usa una herramienta tecnológica como el instrumento de apoyo para la habilidad comunicativa del idioma inglés, al dejar de lado su uso cotidiano y aportar al proceso educativo, una situación desfavorable de esta actividad es que aplica netamente una serie de test para el desarrollo del Speaking, situación que es poco atractiva para la población estudiantil.

Fuente: elaboración propia 
De los anteriores planteamientos, se propone las siguientes fases tales como el Diagnóstico, Planificación, Aplicación y Control, consideradas como los procesos sistemáticos que contribuyen a la destreza de la expresión oral del inglés.

\section{Fase 1. Diagnóstico}

Con la finalidad de diagnosticar el contexto actual de la población estudiantil de básica superior de una de las Unidades Educativas de la zona urbana de Latacunga - Ecuador, se considera relevante la aplicación de una evaluación diagnóstica a 41 estudiantes, la cual a través de la medición de cinco componentes de la expresión oral tales como contenido, pronunciación, fluidez, precisión gramatical y entonación, brinden una pauta para el desarrollo de dicha destreza. Para este fin se utiliza una rúbrica de evaluación de la expresión oral propuesta por Moreno y Ripalda (2015), con una escala Likert, que valora la destreza comunicativa Speaking. Los resultados de la ficha de observación se determinan de la siguiente manera:

\section{Tabla 3}

Porcentajes alcanzados por los estudiantes

\begin{tabular}{llllll}
\hline $\begin{array}{l}\text { Uso de los } \\
\text { Componentes }\end{array}$ & Siempre & Frecuentemente & $\begin{array}{l}\text { Algunas } \\
\text { veces }\end{array}$ & Rara vez & Nunca \\
\hline Contenido & $7,32 \%$ & $14,63 \%$ & $26,83 \%$ & $34,15 \%$ & $17,07 \%$ \\
\hline Pronunciación & $9,76 \%$ & $21,95 \%$ & $31,71 \%$ & $34,15 \%$ & $2,43 \%$ \\
\hline Fluidez & $17,07 \%$ & $17,07 \%$ & $29,27 \%$ & $24,39 \%$ & $12,20 \%$ \\
\hline $\begin{array}{l}\text { Precisión } \\
\text { gramatical }\end{array}$ & $17,07 \%$ & $21,95 \%$ & $17,07 \%$ & $24,39 \%$ & $19,51 \%$ \\
\hline Entonación & $9,75 \%$ & $26,83 \%$ & $21,95 \%$ & $31,71 \%$ & $9,76 \%$ \\
\hline
\end{tabular}

Fuente: elaboración propia

Es evidente que los resultados alcanzados no son favorables, la población evaluada indica que entre los menos aplicados están la precisión gramatical con 19,51\%, el contenido con $17,07 \%$ y la fluidez con 12,20\%, entendiéndose que estos componentes dentro de las prácticas de la oralidad no se desarrollan de manera óptima.

Esto permite a los docentes conocer el nivel de habilidad de sus estudiantes y en relación a ello establecer un trabajo con los educandos.

\section{Fase 2. Planificación}

Para diseñar una estrategia metodológica que mejore la expresión oral del idioma inglés se especifican los participantes y recursos. Como participantes, se encuentran los docentes del área de inglés cuyo distributivo está en los niveles de Básica Superior y por supuesto sus estudiantes, entre los recursos para el diseño de la actividad metodológica están: un ordenador o teléfono móvil con conexión a internet en el aula y el servicio de grabación en línea Vocaroo. 
Para el funcionamiento de Vocaroo, se requiere acceder al sitio web https://s1.vocaroo.com/ o buscarlo directamente en el navegador, los docentes o estudiantes que participarán de este grabador visualizarán lo siguiente:

\section{Figura 1}

Se aprecia la pantalla principal del servicio en línea Vocaroo, la cual se encuentra lista para iniciar un proceso de grabación

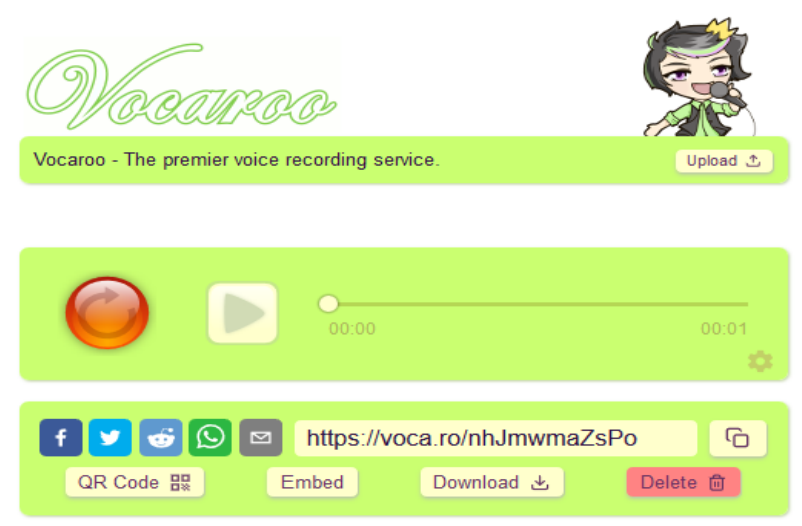

Es necesario indicar que este servicio en línea establece grabaciones inmediatas y sin límite de tiempo, situación que faculta la práctica a favor de la oralidad, el proceso pretende ser atractivo y dinámico y llevarlo más allá que el uso de un grabador normal, es por esto que se sugieren una serie de actividades en las cuales de manera creativa el docente desarrolle la habilidad de expresión oral:

\section{Figura 2}

Se detalla siete actividades que pueden desarrollarse a favor de la habilidad comunicativa del idioma inglés, todas estas aplicables a la herramienta propuesta

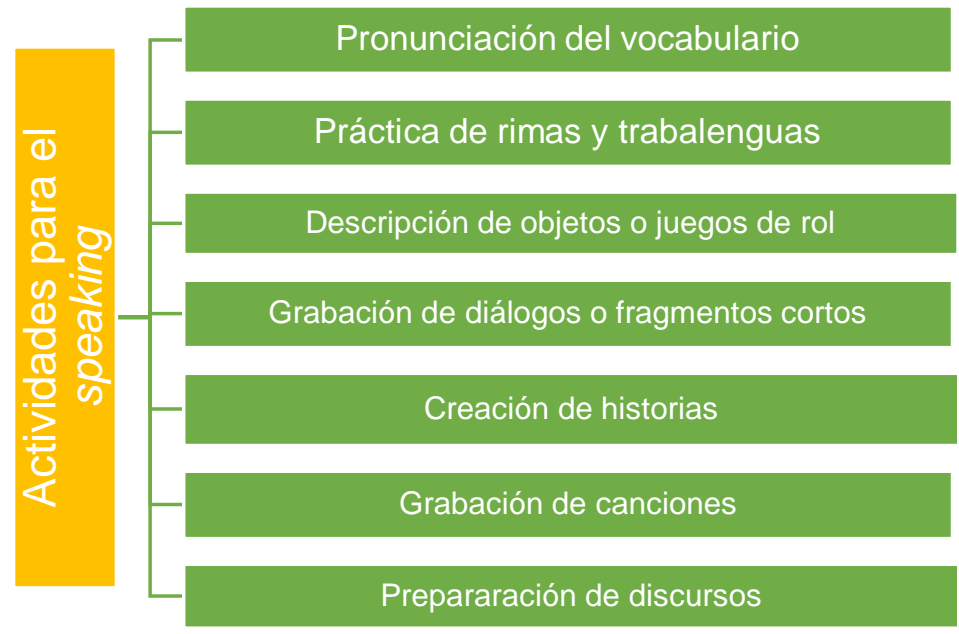


Estas actividades podrían trabajarse en cualquiera de los niveles educativos pero su nivel de complejidad será el variante. En esta oportunidad, se presentará una planificación conforme a básica superior, en donde se ha considerado los contenidos y recursos didácticos del contexto educativo del Ecuador; se toma como referencia el currículo de inglés como lengua extranjera para la habilidad comunicativa en sus respectivos textos con 6 Unidades Didácticas, en donde se pretende alcanzar en los estudiantes un nivel de A1.1, A1.2 e inicio de A2.1, conforme lo propone Ministerio de Educación (2016), para su perfil de salida en el área de lengua extranjera inglés.

\section{Planificación de Actividad}

\section{Tabla 4}

\section{Actividad propuesta}

\section{Unidad 1. PEOPLE AROUND US}

Objetivo: $\quad$ Ser capaz de brindar una introducción sobre el mismo y otros, a través del uso de saludos e información personal en una aplicación tecnológica que provoque una interacción con sus compañeros.

\begin{tabular}{ll}
\hline Participantes: & 25 estudiantes \\
\hline Actividad: & Describir \\
\hline Tiempo: & $\begin{array}{l}\text { En la cuarta semana de la Unidad (Previo desarrollo de contenidos en el } \\
\text { aula). }\end{array}$ \\
\hline Lugar: & Salón de clases \\
\hline $\begin{array}{l}\text { Destrezas con } \\
\text { criterio de }\end{array}$ & $\begin{array}{l}\text { EFL.4.2.16 Inicie, mantenga y finalice una conversación para satisfacer } \\
\text { desempeño }\end{array}$ \\
\hline \multicolumn{2}{l}{ Actividades de Aprendidades básicas y / o maneje una transacción simple. }
\end{tabular}

Individual estudiante

1. Realice una lluvia de ideas con el vocabulario establecido para la actividad.

2. Elabore un guion con las características de la persona a quién describe, omita decir sobre quién se trata.

3. Produce de manera verbal el guion en la aplicación de Vocaroo.

4. Comparte el link al docente través del Código QR.

Docente

5. Recepte los códigos QR.

6. Establezca un intercambio de dichos códigos entre los estudiantes.

Individual estudiante

7. Procure adivinar sobre quién se trata.

8. Socialice al grupo las características que le ayudaron a adivinar de quién se trató.

Recursos: Computador o teléfono móvil, Vocaroo

Técnica: Observación. Instrumento: Ficha de observación

Ejemplo: Canal de YouTube Methodological strategies https://n9.cl/k6m5

Fuente: elaboración propia 
Es importante señalar que, al igual que la actividad planteada se puede trabajar con el resto de temáticas. Dentro de este proceso, el uso de una de las modalidades que contiene la herramienta para compartir en base a Códigos QR (Ver figura 2), brinda un espacio de dinamismo e innovación al proceso de enseñanza aprendizaje en la clase, es por ello que se sugiere usarlo con los estudiantes quienes podrían almacenar sus prácticas en dicha modalidad y compartirlos con sus docentes y compañeros, así también el docente podría imprimir dichos códigos en tal sentido de colocarlos en ciertos espacios del salón de clases y con la ayuda de un lector de códigos en los teléfonos móviles interactuar con las grabaciones, esto transformaría un proceso educativo tradicional, pues, no es muy común observar este tipo de recursos, lo que determina un proceso de aprendizaje lúdico.

\section{Figura 3}

Se presenta el ícono de Códigos QR, el cual se despliega si el usuario comparte a través de esta modalidad de la herramienta Vocarro

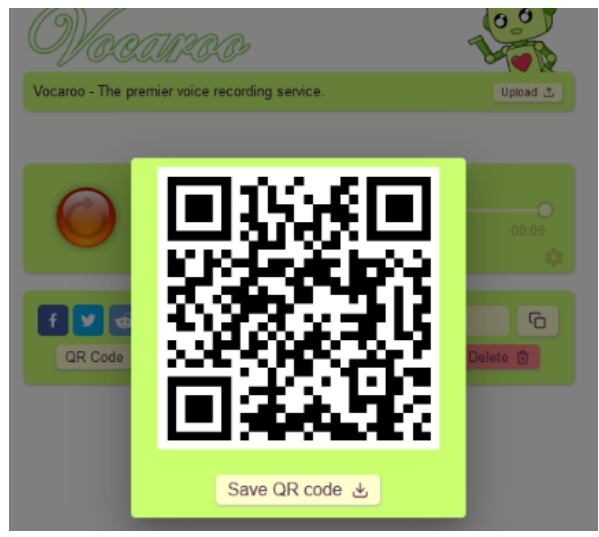

\section{Fase 3. Aplicación}

Se propone a los docentes del área de inglés acceder a las actividades planteadas para la estrategia a través del siguiente canal en YouTube https://n9.cl//k6m5 en donde se encuentra ejercicios para la práctica oral tales como: trabalenguas, narración de historias, diálogos o juegos de roles entre otros.

A continuación, se presenta el procedimiento a seguir:

\section{Tabla 5}

Pasos de aplicación docente

Pasos
1. Seleccione en el canal indicado la actividad a desarrollar.
3. Socialice el proceso participativo a los estudiantes.
4. Solicite se ejecute la actividad de aprendizaje conforme la planificación
del cuadro 5.


5. Se propone una rúbrica para el seguimiento del desarrollo de la habilidad comunicativa.

6. Retroalimente el proceso una vez que los estudiantes efectúen la actividad.

\begin{tabular}{|c|c|c|c|c|c|c|}
\hline \multirow{8}{*}{$\begin{array}{l}\text { Rúbrica de } \\
\text { valoración } \\
\text { (Marca con } \\
\text { una X) }\end{array}$} & \multirow[t]{2}{*}{$\begin{array}{l}\text { Nombre del } \\
\text { estudiante: }\end{array}$} & & & & \multirow{2}{*}{$\begin{array}{c}\text { Fecha: } \\
\text { Muy } \\
\text { bueno } \\
4 \\
\end{array}$} & \multirow[b]{2}{*}{$\begin{array}{c}\text { Excelente } \\
\qquad 5\end{array}$} \\
\hline & & $\begin{array}{c}\text { Escaso } \\
1\end{array}$ & $\begin{array}{c}\text { Justo } \\
2\end{array}$ & $\begin{array}{c}\text { Bueno } \\
\mathbf{3}\end{array}$ & & \\
\hline & Contenido & & & & & \\
\hline & Pronunciación & & & & & \\
\hline & Fluidez & & & & & \\
\hline & $\begin{array}{l}\text { Precisión } \\
\text { gramatical }\end{array}$ & & & & & \\
\hline & Entonación & & & & & \\
\hline & Sugerencia & & & & & \\
\hline
\end{tabular}

Fuente: elaboración propia

\section{Fase 4. Control}

Para el cumplimiento de las actividades propuestas en la estrategia se establece las siguientes acciones:

1. Consolide las rúbricas de valoración para una toma de decisiones en los elementos de la oralidad que presenten dificultad.

2. Establece evaluaciones formativas al final de las unidades para identificar fortalezas y debilidades alcanzadas por los estudiantes.

3. Solicite el criterio de los estudiantes sobre las actividades ejecutadas para la toma de correctivos.

La validación de la estrategia la realizaron los docentes del área de inglés de la institución, el líder Pedagógico y el Rector de la Unidad Educativa, quienes, desde su experticia en las áreas educativas, verifican que la estrategia cuente con condiciones para su aplicación con la población estudiantil, a través de una lista de cotejo institucional, así como, se prevé en un futuro considerar las métricas generadas en las actividades planteadas del canal Methodological strategies en YouTube.

\section{Conclusiones}

El diagnóstico de la situación inicial evidenció un deficiente proceso de desarrollo de la expresión oral, pues entre las escalas de siempre y casi siempre de la totalidad de los 5 componentes se alcanza un 32,68\%, mientras que entre las escalas de algunas veces, rara vez y nunca el porcentaje alcanzado es del $67,32 \%$, esto señala que no es adecuada la participación de 
los estudiantes con esta habilidad, por tanto una estrategia metodológica es necesaria con el fin de potencializar la habilidad comunicativa en el segundo idioma inglés.

Con base a la investigación, se determina que la habilidad comunicativa Speaking, es una de las destrezas imprescindibles por ser el canal que facilita la comunicación entre los países hispanohablantes, por tanto, su proceso de enseñanza aprendizaje requiere estar bien consolidado.

La estrategia metodológica basada en Tecnologías de la Información y Comunicación (TIC's) se crea con la finalidad de aportar a la labor docente e innovar su proceso metodológico a través de la utilización de una herramienta que le permita realizar ejercicios y afianzar los contenidos adquiridos en el salón de clases, se compone de cuatro fases Diagnóstico, Planificación, Aplicación y Control.

\section{Referencias Bibliográficas}

Abio, G. (2014). Grabar audios con Vocaroo y Record MP3 [Plataforma Educativa]. Espacios Santillana Español. https://bit.ly/2ST1vlr

Aldana, Y. (2018). Integración lingüística en la enseñanza del inglés en Colombia mediante el aprendizaje basado en proyectos. Revista Chakiñan de Ciencias Sociales y Humanidades Scielo, 5, 133-145.

Azurra, B. (2015). La importancia de los softwares didácticos en el estudio de lenguas extranjeras en Educación Secundaria Obligatoria [Propuesta teórica]. Universidad Internacional de la Rioja.

Cevallos, B., \& Becerra, E. (2019). Sistema de estrategias metodológicas para el desarrollo del lenguaje oral en los estudiantes del primer nivel de inglés en el centro de idiomas de la Universidad Técnica de Cotopaxi. http://repositorio.uti.edu.ec//handle/123456789/1171

Chacón, C. T., \& Clevia J. Pérez, M. A. (2010). El Podcast como herramienta en la enseñanza del inglés como lengua extranjera. Conocimiento Libre y Educación (CLED), 1(1). http://erevistas.saber.ula.ve/index.php/cled/article/view/279

Costa, R. (2007). Nuevo enfoque metodológico a través de las TIC en el proceso de enseñanzaaprendizaje del inglés: Estrategias de aprendizaje en el entorno virtual. Revista interuniversitaria de formación del profesorado, 59, 183-196.

Education First. (2019). English Proficiency Index 2019, El ranking mundial más grande según su dominio del inglés. Quito: EF EPI. 20.

Gavarri, S. (2016). El aprendizaje de lenguas extranjeras mediado por las TIC: Aprender Inglés con Duolingo. 7, 56-65.

Golkova, D., \& Hubackova, S. (2014). Productive Skills in Second Language Learning. ScienceDirect, 143, 477-481. https://doi.org/10.1016/j.sbspro.2014.07.520

Gonzales, N., Trelles, C., \& Mora, J. (2017). Manejo docente de las Tecnologías de la Información y Comunicación. Cuenca, Ecuador. INNOVA Research Journal, 2(4), 61-72. https://doi.org/10.33890/innova.v2.n4.2017.153 
Harzing, A. (2016). Publish or Perish. Harzing.Com. https://harzing.com/resources/publish-orperish

Madero, F. (2018). La Habilidad de Speaking en Inglés, una propuesta Lúdica para su Desarrollo. Fundación Universitaria Los Libertadores, 60.

Maldonado, K., Vera, R., Ponce, L., \& Toala, F. (2020). Software educativo y su importancia en el proceso enseñanza-Aprendizaje. UNESUM-Ciencias. Revista Científica Multidisciplinaria. ISSN 2602-8166, 4(1), 123-130.

Marín, C. (2009). Manual de Estrategias de Aprendizaje del idioma inglés. Universidad Veracruzana - Centro de Auto-acceso Coatzacoalcos.

Martínez, A. L. (2020). Estrategias metodológicas para mejorar las habilidades de speaking y listening en idioma ingles de acuerdo a estilos de aprendizaje. Con-Ciencia Serrana Boletín Científico de la Escuela Preparatoria Ixtlahuaco, 2(4), 33-35.

Ministerio de Educación. (2014). Acuerdo $N^{\circ} 052-14$. Normativa para la implementación e instrumentalización de la disposición transitoria contenida en el acuerdo ministerial No. 041-14.

Ministerio de Educación. (2016). Currículo de los niveles de Educación Obligatoria Subnivel Superior-Lengua Extranjera (https://educacion.gob.ec/curriculo-superior/).

Morales, M., Benítez, C., Silva, D., Altamirano, M., \& Mendoza, H. (2015). Aplicación móvil para el aprendizaje del inglés utilizando realidad aumentada. 2, 18.

Moreno, M., \& Ripalda, M. (2015). Diseño de una estrategia comunicativa en nivel Al de Inglés del MCER para el desarrollo de la expresión oral en Educación Básica Superior. https://repositorio.pucesa.edu.ec/handle/123456789/1363

Moreno, N., Leiva, J., \& Matas, A. (2016). Mobile learning, Gamificación y Realidad Aumentada para la enseñanza-aprendizaje de idiomas. IJERI: International Journal of Educational Research and Innovation, 6, 16-34.

Norato, N., \& López, D. (2018, septiembre 6). Aprender inglés tiene su ciencia: Una estrategia Pedagógica encaminada al Bilingüismo. Congreso Internacional de Educaciones, pedagógicas y Didácticas. Congreso Internacional de Educaciones, pedagógicas y Didácticas. https://bit.ly/34VPnpl

Nunan, D. (1991). Language Teaching Methodology. A textbook for teachers. Prentice Hall International English Language Teaching.

Núñez, S., \& Medina, P. (2020). Teaching English language grammar seen through an inverted classroom. 5(19).

Ochoa, M., García, M., Hernández, M., \& Navarro, M. (2016). El uso de Estrategias para el Aprendizaje de vocabulario Académico en el Idioma Inglés en el Nivel Superior. Educateconciencia,

$7(8)$. http://tecnocientifica.com.mx/educateconciencia/index.php/revistaeducate/article/view/41

Pacheco, J., \& Barros, J. (2013). O Uso de Softwares Educativos no Ensino de Matemática. Revista Diálogos, 8, 5-13.

Pizarro, G., \& Cordero, D. (2013). Las TIC: Una herramienta tecnológica para el desarrollo de las competencias lingüísticas en estudiantes universitarios de una segunda lengua. Revista Electrónica Educare, 17, 16. 
Ricoy, M., \& Álvarez, S. (2016). La enseñanza del inglés en la educación básica de personas jóvenes y adultas. Revista mexicana de investigación educativa, 21(69), 385-409.

Toscano, C., \& Fonseca, M. (2013). La música como herramienta facilitadora del aprendizaje del inglés como lengua extranjera. Teoría de la Educación. Revista Interuniversitaria, 24(2), 197-213-213. https://doi.org/10.14201/teri.10361

Utrera, E. (2012). Juegos para aprender inglés (1era ed.). https://bit.ly/3dpmaqD

Vélez, P., \& Yaguana, Y. (2019). New technologies in the teaching learning process (RiUTPL). Studia Humanitatis, Universidad Técnica Particular de Loja. https://bit.ly/3nMIv6h

Vergara, A., \& Perdomo, M. (2017). Fortalecimiento de la expresión oral y escrita en inglés a través de un andamiaje de escritura creativa colaborativa: Un estudio de diseño desde la cognición distribuida. Forma y Función, 30(1), 117-155. https://doi.org/10.15446/fyf.v30n1.62417

Vidal, M., Gómez, F., \& Ruiz, A. (2010). Software educativos. Educación Médica Superior, 24(1), 97-110.

Yorca, M., González, Y., \& Garcés, G. (2019). El uso de las tecnologías de la información y la comunicación en el aprendizaje del idioma inglés en la Universidad de Las Tunas. Opuntia Brava, 11(Especial 1), 282-291. https://doi.org/10.35195/ob.v11iEspecial.684

Yumi, L., Padilla, N., Padilla, Y., \& Obregon, Á. (2020). Mejoramiento del Speaking en el idioma inglés con el uso de WhatsApp. Revista ESPACIOS, 41. 